\title{
Design and implementation of an efficient WPT system
}

\author{
Abdulkareem Mokif Obais ${ }^{1}$, Ali Faeq Ruzij ${ }^{2}$ \\ ${ }^{1}$ Department of Biomedical Engineering, University Babylon, Iraq. \\ ${ }^{2}$ Department of Electrical Engineering, University of Babylon, Iraq.
}

\begin{tabular}{l}
\hline Article Info \\
\hline Article history: \\
Received Oct 10, 2019 \\
Revised Nov 9, 2019 \\
Accepted Jan 22, 2020 \\
\hline
\end{tabular}

\section{Keywords:}

Efficiency enhancement

Inductive link

Mutual coupling

Mutual inductance

Wireless power transfer

\begin{abstract}
Wireless power transfer (WPT) is a technique introduced to transfer power wirelessly. Generally, WPT systems are characterized by low efficiency and low output power. Since WPT process depends mainly on mutual coupling between transmitting and receiving coils in addition to load requirements, it is focused in this work toward enhancing the mutual coupling and conditioning the receiving circuit so as to optimally satisfy the load demand. The mutual coupling between transmitting and receiving nodes is enhanced via inserting three resonating circuits along with energy transmission path and conditioning the receiving circuit such that it accomplishes delivering maximum power to the load node. In this work, an adaptive efficient WPT system is introduced. This system is carried out on PSpice and validated experimentally. Both simulative and experimental WPT systems have accomplished significant enhancement in efficiency. The proposed WPT system has three resonators and three parallel connected identical receiving coils located at $6.61 \mathrm{~m}$ from the power transmitter. The efficiency enhancement approaches thousands of times the efficiency of a conventional WPT system having similar power transmitter located at the same distance from the receiving circuit, which has a single coil identical to those in the proposed efficient WPT system.
\end{abstract}

This is an open access article under the CC BY-SA license.

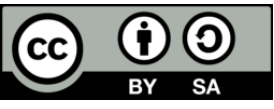

\section{Corresponding Author:}

Abdulkareem Mokif Obais,

Department of Biomedical Engineering,

University of Babylon,

Hilla, Babylon, Iraq.

Email: karimobais@yahoo.com

\section{INTRODUCTION}

In the last years, the enhancing technology of power transfer (WPT) develops quickly. The mobiles or other electronic devices such as laptops or electrical vehicles could be charged by WPT technology. These techniques could be used in places when wiring is difficult to be accomplished [1-5]. Long-range concept of WPT had been formulated after microwave amplifier invention for high power purposes. The possibility of power transmission via electromagnetic waves gained more and more intention for application purposes $[6,7]$. The works curried out by $[7,8]$ present analytical models for calculating the mutual inductance between planar spiral coils. These models are very useful in the design and optimization of wireless power transfer systems. The proposed models of mutual inductances between coils were derived using the solution of Neumann's integral. An approximate formula for determining the self-resistance of a circular multi-loop inductor having unequal pitches was introduced by [9]. Both proximity and skin effects were included in the suggested formula. Proximity effect was determined using the magnetic fields exerted on a wire due to other wires.

The study conducted by [10] analyzed with a context of non-ferromagnetic metallic plate, the WPT performances. The model of the WPT impedance in the metal environment was built. The proposed system in [11] comprised several loop inductors having different sizes. Depending on the changes of the distance 
between the transmitting and receiving sides, the power was switched to one loop of the inductors for power transmission and power reception.

A WPT system of $13.56 \mathrm{MHz}$ was introduced by [12] and investigated both theoretically and experimentally in addition to simulation. A relative high improvement in efficiency of about $41.7 \%$ was achieved. Three phase angles had been designed and analyzed in [13]. Single power amplifier on the primary side and two power amplifiers were located at the secondary side. The proposed multi-degrees method of phase control was capable of achieving simultaneously additional compensation of the reactance, output regulation, and load transformation.

A consistent optimization technique was conducted by [14] for WPT systems equipped with passive element's enhancement beginning from simple reflectors, intermediate relays, and general electromagnetic focusing and guiding structures, like metamaterials and metasurfaces. The proposed work efficiently solved the problem of optimization using arbitrary numbers of passive elements.

The work conducted by [15] presented and explained the published techniques and principles concerning all aspects of inductive link design processes such that no specific preceding information about inductive link designs are required. The work in [16] introduced accurate formulas for calculating mutual inductance between spiral coils using Gaussian integration method. The researches introduced by [17-22] concern enhancing coupling coefficients in different environments of inductive links in addition to applications of WPT technologies in contactless electrical vehicles and energizing medical sensors.

In this work, poor efficiency of conventional wireless powering process is enhanced by strengthening the mutual coupling along with power transmission path and conditioning the receiving circuit such that it accomplishes maximum power reception to load node.

\section{Design of the proposed efficient WPT system}

The inductive mutual coupling $M_{a b}$ between two 1-turn planar spiral coils is shown in Figure 1a. The mutual inductance between these two coils is given by [7]

$$
\begin{aligned}
& M_{a b}=\frac{\mu_{0} \pi a^{2} b^{2}}{\left(a^{2}+b^{2}+z^{2}\right)^{3 / 2}}\left(1+\frac{15}{32} \gamma^{2}+\frac{315}{1024} \gamma^{4}\right) \\
& \gamma=\frac{2 a b}{a^{2}+b^{2}+z^{2}}
\end{aligned}
$$

Where, $a$ and $b$ are the outer radii of the first and second coils, respectively. $\mu_{0}$ is the permeability of free space and $z$ is the distance between the centers of the two coils, which are aligned coaxially.

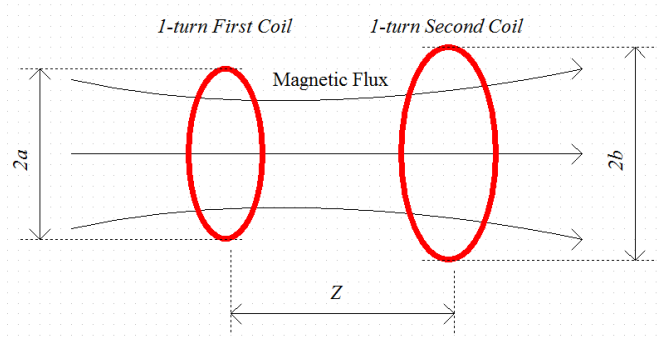

(a)

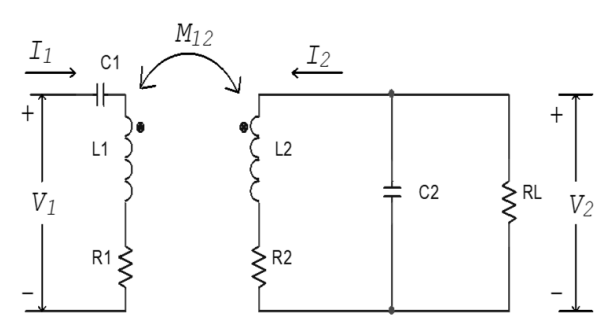

(b)

Figure 1. Inductive link, (a) coupling between two 1-turn coils, (b) modeling of two coil inductive link.

For multi-turns planar spiral coils, (1) can be modified to

$$
\begin{aligned}
& M_{12}=\sum_{i=1}^{N_{1}} \sum_{j=1}^{N_{2}} \frac{\mu_{0} \pi a_{i}^{2} b_{j}^{2}}{\left(a_{i}^{2}+b_{j}^{2}+z^{2}\right)^{3 / 2}}\left(1+\frac{15}{32} \gamma_{i j}^{2}+\frac{315}{1024} \gamma_{i j}^{4}\right) \\
& \gamma_{i j}=\frac{2 a_{i} b_{j}}{a_{i}^{2}+b_{j}^{2}+z^{2}}
\end{aligned}
$$


Where, $N_{1}$ and $N_{2}$ are the numbers of turns of the first and second coils, respectively. If two inductively coupled coils are forming a resonant inductive link shown in Figure 1b, then the link currents and voltages at resonance frequency can be given by

$$
\begin{aligned}
& I_{1}=\frac{V_{1}}{R_{1}+\frac{\left(\omega_{0} M_{12}\right)^{2}}{R_{2}}}=\frac{V_{1}}{R_{1}+R_{\text {refl }}}, R_{L} \gg \frac{1}{\omega_{0} C_{2}} \\
& I_{2}=-\frac{j \omega_{0} M_{12} V_{1}}{R_{1} R_{2}+\left(\omega_{0} M_{12}\right)^{2}}, R_{L} \gg \frac{1}{\omega_{0} C_{2}}
\end{aligned}
$$

Where, $L_{1}$ and $L_{2}$ are self-inductances of the first and second coils, respectively. $M_{12}$ is the mutual inductance between the two coils. $R_{1}$ and $R_{2}$ are the AC resistances of the first and second coils, respectively. $C_{1}$ and $C_{2}$ are the tuning capacitances of the sending (transmitting) and receiving circuits, respectively, whereas $\omega_{0}=2 \pi f_{0}$ is the inductive link operating angular frequency. $R_{\text {refl }}$ is defined by

$$
R_{\text {refl }}=\frac{\left(\omega_{0} M_{12}\right)^{2}}{R_{2}}
$$

The output voltage $V_{2}$ is determined by

$$
V_{2}=-I_{2} \frac{1}{j \omega_{0} C_{2}}=\frac{\omega_{0}^{2} M_{12} L_{2} V_{1}}{R_{1} R_{2}+\left(\omega_{0} M_{12}\right)^{2}}, R_{L} \gg \frac{1}{\omega_{0} C_{2}},\left(\frac{1}{\omega_{0} C_{2}}=\omega_{0} L_{2}\right)
$$

The link input power $P_{i}$, output power $P_{0}$, and efficiency $\eta$ can be given by

$$
\begin{aligned}
& P_{i}=\frac{\left|V_{1}\right|^{2}}{R_{1}+\frac{\left(\omega_{0} M_{12}\right)^{2}}{R_{2}}}, R_{L} \gg \frac{1}{\omega_{0} C_{2}} \\
& P_{0}=\frac{\left|V_{2}\right|^{2}}{R_{L}}=\frac{1}{R_{L}}\left(\frac{\omega_{0}^{2} M_{12} L_{2} V_{1}}{R_{1} R_{2}+\left(\omega_{0} M_{12}\right)^{2}}\right)^{2}, R_{L} \gg \frac{1}{\omega_{0} C_{2}} \\
& \eta=\frac{P_{0}}{P_{i}}=\frac{Q_{1} Q_{2}^{2} k_{12}^{2}}{\omega_{0} L_{2} Q_{L}\left(1+Q_{1} Q_{2} k_{12}^{2}\right)}, R_{L} \gg \frac{1}{\omega_{0} C_{2}}
\end{aligned}
$$

Where, $Q_{1}, Q_{2}$, and $Q_{L}$ are the quality factors of the first coil, second coil, and load, respectively at resonance frequency $f_{0} . Q_{1}, Q_{2}$, and $Q_{L}$ are defined by $\omega_{0} L_{1} / R_{1}, \omega_{0} L_{2} / R_{2}$, and $R_{L} / \omega_{0} L_{2}$, respectively. $k_{12}$ is the coefficient of coupling between the two coils and is defined by

$$
k_{12}=\frac{M_{12}}{\sqrt{L_{1} L_{2}}}
$$

For loosely coupled inductive link (11) can closely be approximated to

$$
\eta=\frac{Q_{1} Q_{2}^{2} k_{12}^{2}}{\omega_{0} L_{2} Q_{L}}, R_{L} \gg \frac{1}{\omega_{0} C_{2}} \text { and } Q_{1} Q_{2} k_{12}^{2} \ll 1
$$

According to (11), the efficiency of WPT process is directly proportional to the square of the coupling coefficient. The proposed efficient WPT system can be depicted by block diagram of Figure 2 .

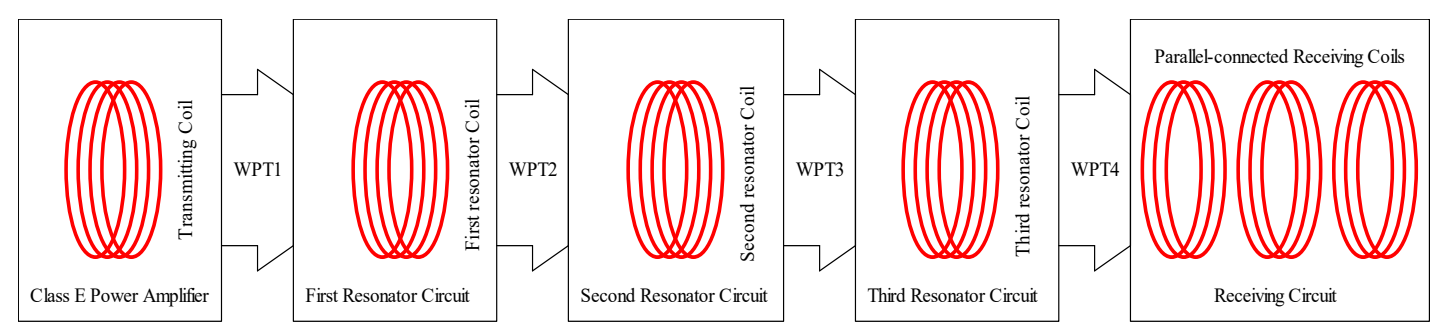

Figure 2. The block diagram of the proposed system. 
It is obvious that the proposed system shown in Figure 2 comprises four series WPT systems. In this work, three resonating coils are inserted on the path joining the transmitting and receiving coils. The locations of resonating coils are selected such that maximum power is transferred between each two adjacent coils. Figure 3 shows the alignment of coils in the proposed inductive link. The first coil from the left is $L_{1}$, which represents the transmitting coil and its distance $z_{12}$ from the second coil $L_{2}$ (first resonator coil) is $156 \mathrm{~cm}$. At $227 \mathrm{~cm}$ to the right of the first resonator is located the third coil $L_{3}$ (second resonator coil), whereas the fourth coil $L_{4}$ (third resonator coil) is located at $140 \mathrm{~cm}$ to right of the second resonator. Three similar receiving coils $L_{5}, L_{6}$, and $L_{7}$ are oriented $50 \mathrm{~cm}$ to the right of the third resonator and each adjacent coils are $20 \mathrm{~cm}$ apart.

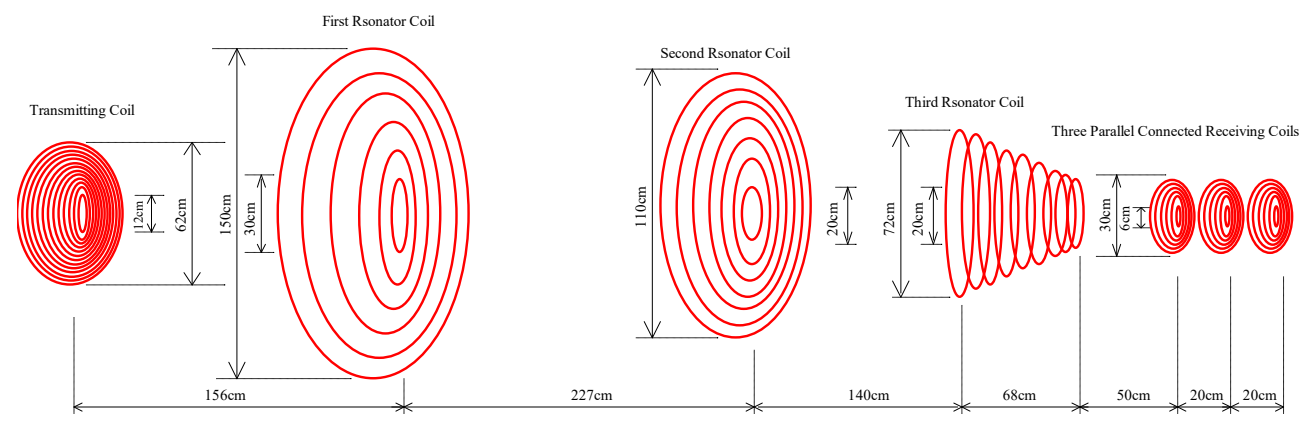

Figure 3. Alignment of coils in the proposed system.

The transmitting coil is shown in Figure 4a. It is wound with 12.667 turn's copper pipe having an outer diameter of $19.05 \mathrm{~mm}$ and wall thickness of $0.91 \mathrm{~mm}$. The coil outer diameter is $62 \mathrm{~cm}$, whereas its inner diameter is $12 \mathrm{~cm}$. The first resonator shown in Figure $4 \mathrm{~b}$ is a planar spiral coil wound with 5.5 turn's copper pipe having outer diameter of $19.05 \mathrm{~mm}$ and wall thickness of $0.91 \mathrm{~mm}$. The coil outer and inner diameters are $150 \mathrm{~cm}$ and $30 \mathrm{~cm}$, respectively. The second resonator shown in Figure $4 \mathrm{c}$ is a planar spiral coil wound with 7.25 turn's copper pipe having outer diameter of $6.35 \mathrm{~mm}$ and wall thickness of $0.71 \mathrm{~mm}$. The coil outer diameter is $110 \mathrm{~cm}$ and its inner diameter is $20 \mathrm{~cm}$. The third resonator shown in Figure $4 \mathrm{~d}$ is a conical coil wound with 9.25 turn's copper pipe, which has an outer diameter of $19.05 \mathrm{~mm}$ and wall thickness of $0.91 \mathrm{~mm}$. The coil outer and inner diameters are $72 \mathrm{~cm}$ and $20 \mathrm{~cm}$, respectively. The coil length is $68 \mathrm{~cm}$.

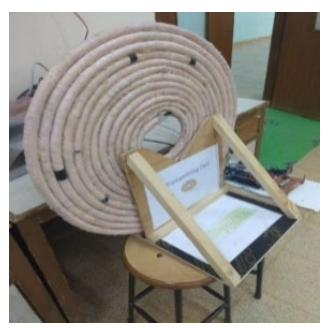

(a)

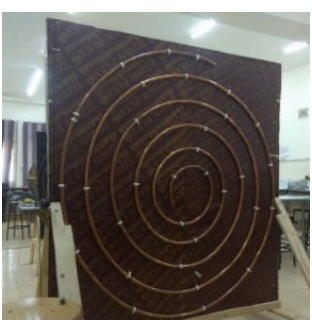

(b)

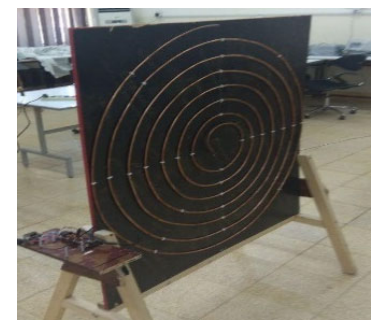

(c)

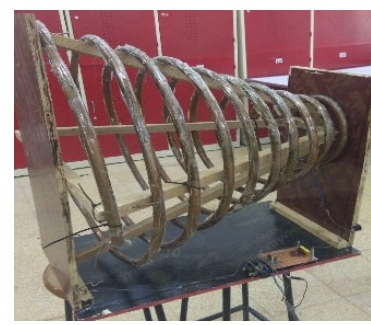

(d)

Figure 4. Photos of (a) the transmitting coil $\left(L_{1}\right)$, (b) the first resonator coil $\left(L_{2}\right)$, (c) the second resonator coil $\left(L_{3}\right)$, (d) the third resonator coil $\left(L_{4}\right)$.

The receiving circuit is shown in Figure 5. It is composed of three similar planar spiral coils connected in parallel. Each coil is wound with 6 turn's copper pipe having outer diameter of $6.35 \mathrm{~mm}$ and wall thickness of $0.71 \mathrm{~mm}$. The coil outer and inner diameters are $33.5 \mathrm{~cm}$ and $6 \mathrm{~cm}$, respectively. The coils in the proposed WPT system are wound with pipe conductors in order to decrease their AC resistances by decreasing the skin effect, which has great impact on the quality factors of coils. Decreasing the AC resistances of coils increases the coils quality factor, which in turn increases the link efficiency governed by (11) or (13), which is applicable for loosely coupled inductive links.

Int J Pow Elec \& Dri Syst, Vol. 11, No. 2, June 2020 : $711-725$ 
The proposed WPT system depicted in Figure 2 can be schematically represented by the circuit shown Figure 6. In this Figure, $R_{1}, R_{2}, R_{3}, R_{4}, R_{5}, R_{6}$, and $R_{7}$ are the AC resistances of the coils $L_{1}, L_{2}, L_{3}, L_{4}$, $L_{5}, L_{6}$, and $L_{7}$, respectively. $C_{1}, C_{2}, C_{3}, C_{4}$, and $C_{5}$ are tuning capacitors of transmitting, first resonator, second resonator, third resonator, and receiving circuits, respectively. Each $M$ represents the mutual inductance between two certain coils. For example $M_{12}$ represents the mutual inductance between $L_{1}$ and $L_{2}$. $M_{12}$ is equal to $M_{21}\left(M_{12}=M_{21}\right)$ and this is applicable for each two coils.

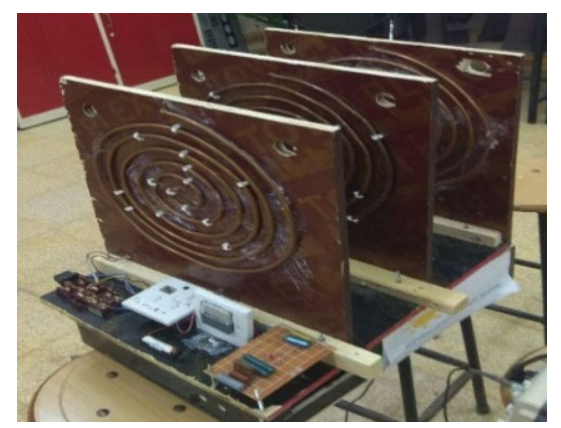

Figure 5. A photo of the receiving circuit, which is composed of three planar spiral coils $\left(L_{5}, L_{6}\right.$, and $\left.L_{7}\right)$.

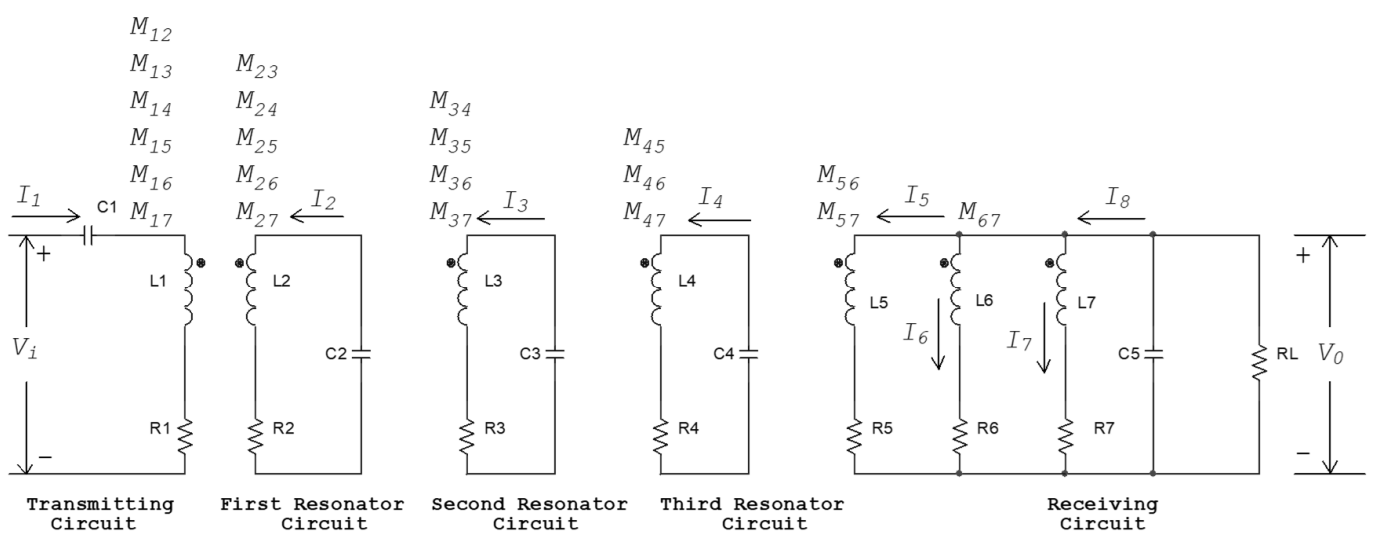

Figure 6. Schematic representation of the proposed system.

The inductances of planar spiral coils can be calculated by [15]

$$
L_{\text {Spiral }}=\frac{\mu_{0} n^{2} d_{\text {avg }}}{2}\left(\ln \left(\frac{2.46}{\beta}\right)+0.2 \beta^{2}\right)
$$

Where, the average diameter is denoted by $d \mathrm{avg}=0.5\left(d_{o}+d_{\mathrm{i}}\right) . d_{i}$ and $d_{0}$ are the inner and outer diameters of the coil, respectively, while $\beta$ is the fill-factor, which is given by

$$
\beta=\frac{d o-d \mathrm{i}}{d o+d \mathrm{i}}
$$

The inductance of the conical coil shown in Figure 7 can be calculated as follows [23]:

$$
L_{\text {con }}=\frac{0.314 n^{2}\left(d_{i}+\operatorname{sncos} Y\right)^{2}}{4 d_{i}+\operatorname{sn}(4 \cos Y+11)},(\mu H)
$$

Where, $n, d_{i}, s$, and $Y$ are coil number of turns, inner diameter, distance between two adjacent turns, and coil angle, respectively. $Y$ and $s$ are defined by 


$$
\begin{aligned}
& Y=\tan ^{-1} \frac{l}{0.5\left(d_{0}-d_{i}\right)} \\
& S=\frac{W}{n}
\end{aligned}
$$

Where, $d_{0}$ is the coil outer diameter. $W$ and $l$ are coil axial and longitudinal lengths, respectively. All dimensions are in inches. Substituting for $\mathrm{n}=9.25, d_{i}=20 \mathrm{~cm}=7.874$ inches, $d_{0}=72 \mathrm{~cm}=28.346$ inches, $l=68 \mathrm{~cm}=26.772$ inches, and $W=72.8 \mathrm{~cm}=28.661$ inches into (16) to (18) gives $L_{c o n}=22 \mu \mathrm{H}$.

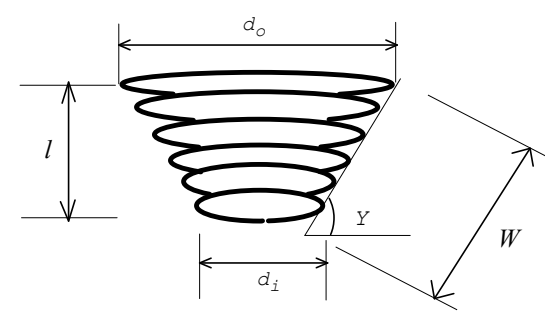

Figure 7. The inverse conical coil.

The DC resistances of all coils in the proposed WPT system can be calculated by

$$
R_{D C}=\frac{l_{C}}{\pi \sigma_{C u}\left(\left(\frac{w}{2}\right)^{2}-\left(\frac{w}{2}-w_{t h}\right)^{2}\right)}
$$

Where, $l_{C}, \sigma_{C u}, w$, and $w_{t h}$, are the coil length, conductivity of copper, outer diameter of coil pipe conductor, and wall thickness of the pipe. The calculated inductances and DC resistances are listed in Table 1. The mutual inductances between coils can be calculated using (3). The coefficients of coupling between coils are calculated according to (12). The calculated mutual inductances and their corresponding coefficients of coupling are listed in Table 2.

Table 1. Coil's calculated DC resistance and inductances

\begin{tabular}{ccc}
\hline Coil Name & DC Resistance $(\Omega)$ & Inductance $(\mu \mathrm{H})$ \\
\hline$L_{1}$ & 0.0041827 & 46.334 \\
$L_{2}$ & 0.00448 & 23.864 \\
$L_{3}$ & 0.018474 & 29.3 \\
$L_{4}$ & 0.0039 & 22 \\
$L_{5}, L_{6}, L_{7}$ & 0.00458 & 6.0745 \\
\hline
\end{tabular}

The AC resistance $R_{A C}$ of a coil wound with copper conductors can be calculated by [24]

$$
\begin{aligned}
& R_{A C}=R_{D C} \frac{\frac{w}{2}}{2 \delta_{C u}} \\
& \delta_{C u}=\frac{1}{\sqrt{\mu_{0} \pi \sigma_{C u} f_{0}}}
\end{aligned}
$$

Where, $R_{D C}, \sigma_{C u}, w$, and $\delta_{C u}$ are the DC resistance of the coil, conductivity of copper, outer diameter of the coil conductor, and copper skin depth at $f_{0}$. Respectively.

Applying (20) using the conductor outer diameters and calculated DC resistance in Table 1, the AC resistances of all coils are calculated and listed in Table 3. 
Table 2. Mutual inductances and their corresponding coupling

Table 3. Coil's calculated AC resistances coefficients

\begin{tabular}{cccc}
\hline $\begin{array}{c}\text { Mutual } \\
\text { Inductance }\end{array}$ & Value $(\mu \mathrm{H})$ & $\begin{array}{c}\text { Coefficient of } \\
\text { Coupling }\end{array}$ & Value \\
\hline$M_{12}$ & 0.2621 & $k_{12}$ & 0.007882 \\
$M_{13}$ & 0.0161 & $k_{13}$ & 0.000437 \\
$M_{14}$ & 0.003 & $k_{14}$ & 0.000094 \\
$M_{15}$ & 0.000281 & $k_{15}$ & 0.00001675 \\
$M_{16}$ & 0.0002564 & $k_{16}$ & 0.0000154 \\
$M_{17}$ & 0.0002345 & $k_{17}$ & 0.00001295 \\
$M_{23}$ & 0.1592 & $k_{23}$ & 0.006 \\
$M_{24}$ & 0.0179 & $k_{24}$ & 0.000781 \\
$M_{25}$ & 0.0015 & $k_{25}$ & 0.0001246 \\
$M_{26}$ & 0.0013 & $k_{26}$ & 0.000108 \\
$M_{27}$ & 0.0012 & $k_{27}$ & 0.0001 \\
$M_{34}$ & 0.1358 & $k_{34}$ & 0.00535 \\
$M_{35}$ & 0.007 & $k_{35}$ & 0.000528 \\
$M_{36}$ & 0.0057 & $k_{36}$ & 0.000427 \\
$M_{37}$ & 0.0046 & $k_{37}$ & 0.0003448 \\
$M_{45}$ & 0.1931 & $k_{45}$ & 0.0167 \\
$M_{46}$ & 0.0985 & $k_{46}$ & 0.00852 \\
$M_{47}$ & 0.0558 & $k_{47}$ & 0.004827 \\
$M_{56}$ & 0.5018 & $k_{56}$ & 0.083633 \\
$M_{57}$ & 0.1161 & $k_{57}$ & 0.01935 \\
$M_{67}$ & 0.5018 & $k_{67}$ & 0.083633 \\
\hline & & &
\end{tabular}

\begin{tabular}{ccc}
\hline $\begin{array}{c}\text { Coil } \\
\text { Name }\end{array}$ & $\begin{array}{c}\text { AC } \\
\text { Resistance }\end{array}$ & $\begin{array}{c}\text { AC Resistance } \\
\text { Value }(\Omega)\end{array}$ \\
\hline$L_{1}$ & $R_{1}$ & 0.185 \\
$L_{2}$ & $R_{2}$ & 0.2 \\
$L_{3}$ & $R_{3}$ & 0.27246 \\
$L_{4}$ & $R_{4}$ & 0.172 \\
$L_{5}$ & $R_{5}$ & 0.0676 \\
$L_{6}$ & $R_{6}$ & 0.0676 \\
$L_{7}$ & $R_{7}$ & 0.0676 \\
\hline
\end{tabular}

Since the transmitting coil $\left(L_{1}\right)$ and the three resonating coils $\left(L_{2}, L_{3}\right.$, and $\left.L_{4}\right)$ are operating within loosely coupled conditions, then the tuning capacitors $C_{2}, C_{3}$, and $C_{4}$ shown in Figure 6 can be determined as $9.25 \mathrm{nF}, 7.5345 \mathrm{nF}$, and $10.034463 \mathrm{nF}$, respectively. The circuit elements $C_{l}$ and $L_{I}$ in Figure 6 are parts of the output circuit of the class-E power amplifier, which represents the power transmitter of the proposed efficient WPT system. The series combination formed by $C_{l}$ and $L_{l}$ Are designed to resonate at a frequency slightly less than $f_{0}$ and their equivalent impedance at the resonance frequency $f_{0}$ is $R_{I}+j X$, where $X$ is defined by [25]

$$
X=1.152 R=\omega_{0} L_{1}-\frac{1}{\omega_{0} C_{1}}
$$

Where, $R$ represents the resistive load of class-E power amplifier and can be determined by

$$
R=R_{1}+R_{\text {refl }}
$$

The tuning capacitor $C_{5}$ of the receiving coils shown in Figure 6 can be approximately determined in a different procedure. Figure 8 models the receiving coils without $C_{5}$ and $R_{L}$. Since the receiving circuit is symmetrical around the branch including $L_{6}$, then $I_{5}=I_{7}$ and $I_{6}=-2 I_{5}$. Consequently, the impedance $Z_{R C V}$ of the parallel-connected receiving coils can be determined by

$$
Z_{R V C}=\left(0.5 R_{5}+j 0.5 \omega_{0} L_{5}-j \omega_{0} M_{56}+j 0.5 \omega_{0} M_{57}\right) / /\left(R_{5}+j \omega_{0} L_{5}+j 2 \omega_{0} M_{56}\right)
$$

Substituting the calculated circuit parameters in (24) gives

$$
Z_{R V C}=0.023+j 4.04, \Omega
$$

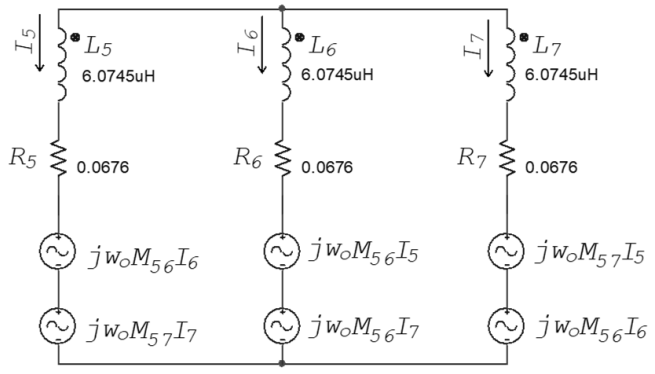

Figure 8. Modeling of the receiving coils without $C_{5}$ and $R_{L}$. 
According to (25), the required value for $C_{5}$ to accomplish resonance is $116.3 \mathrm{nF}$. At resonance frequency $f_{0}$ and after tuning all capacitors such that all link circuits resonate at the same time, the link currents can be related to each other by

$$
\left[\begin{array}{c}
V_{i} \\
0 \\
0 \\
0 \\
0 \\
0 \\
0
\end{array}\right]=\left[\begin{array}{ccccccc}
R_{1}+j X & j \omega_{0} M_{12} & j \omega_{0} M_{13} & j \omega_{0} M_{14} & j \omega_{0} M_{15} & j \omega_{0} M_{16} & j \omega_{0} M_{17} \\
j \omega_{0} M_{21} & R_{2} & j \omega_{0} M_{23} & j \omega_{0} M_{24} & j \omega_{0} M_{25} & j \omega_{0} M_{26} & j \omega_{0} M_{27} \\
j \omega_{0} M_{31} & j \omega_{0} M_{32} & R_{3} & j \omega_{0} M_{34} & j \omega_{0} M_{35} & j \omega_{0} M_{36} & j \omega_{0} M_{37} \\
j \omega_{0} M_{41} & j \omega_{0} M_{42} & j \omega_{0} M_{43} & R_{4} & j \omega_{0} M_{45} & j \omega_{0} M_{46} & j \omega_{0} M_{47} \\
j \omega_{0} M_{51} & j \omega_{0} M_{52} & j \omega_{0} M_{53} & j \omega_{0} M_{54} & Z_{55} & Z_{56} & Z_{57} \\
j \omega_{0} M_{61} & j \omega_{0} M_{62} & j \omega_{0} M_{63} & j \omega_{0} M_{64} & Z_{65} & Z_{66} & Z_{67} \\
j \omega_{0} M_{71} & j \omega_{0} M_{72} & j \omega_{0} M_{73} & j \omega_{0} M_{74} & Z_{75} & Z_{76} & Z_{77}
\end{array}\right]\left[\begin{array}{l}
I_{1} \\
I_{2} \\
I_{3} \\
I_{4} \\
I_{5} \\
I_{6} \\
I_{7}
\end{array}\right]
$$

Where, $I_{1}, I_{2}, I_{3}, I_{4}, I_{5}, I_{6}$, and $I_{7}$ are the currents flowing through coils $L_{1}, L_{2}, L_{3}, L_{4}, L_{5}, L_{6}$, and $L_{7}$, respectively. Since, $L_{l}$ and $C_{l}$ are parts of the class-E driving power amplifier and resonate at a frequency slightly less than the link resonance frequency $f_{0}$, the inductive reactance $X$ represents their equivalent reactance at $f_{0 .} Z_{55}, Z_{56}, Z_{57}, Z_{65}, Z_{66}, Z_{67}$, $Z_{75}, Z_{76}$, and $Z_{77}$ are defined by

$$
\left[\begin{array}{lll}
Z_{55} & Z_{56} & Z_{57} \\
Z_{65} & Z_{66} & Z_{67} \\
Z_{75} & Z_{76} & Z_{77}
\end{array}\right]=\left[\begin{array}{ccc}
R_{5}+j \omega_{0} L_{5}+\frac{1}{j \omega_{0} C_{5}} & j \omega_{0} M_{56}+\frac{1}{j \omega_{0} C_{5}} & j \omega_{0} M_{57}+\frac{1}{j \omega_{0} C_{5}} \\
M_{65}+\frac{1}{j \omega_{0} C_{5}} & R_{6}+j \omega_{0} L_{6}+\frac{1}{j \omega_{0} C_{5}} & M_{67}+\frac{1}{j \omega_{0} C_{5}} \\
M_{75}+\frac{1}{j \omega_{0} C_{5}} & M_{76}+\frac{1}{j \omega_{0} C_{5}} & R_{7}+j \omega_{0} L_{7}+\frac{1}{j \omega_{0} C_{5}}
\end{array}\right]
$$

According to the block diagram of the overall proposed WPT system, there are four series WPT systems namely WPT1, WPT2, WPT3, and WPT4. The first three systems WPT1, WPT2, and WPT3 are loosely coupled systems due to the relatively large distances between their transmitting and receiving coils, thus the first WPT system (WPT1) can be analyzed separately from other systems. Figure 9 shows the model of the resonant inductive link corresponding to WPT1 system. At resonance frequency $f_{0}$, the reflected resistance $R_{\text {refl }}$ from the secondary side (first, second, and third resonators) in the primary side (transmitting coil) is determined according to Equation (7) as $1.56 \Omega$. $V^{\prime}$ in Figure 9 represents the voltage across the resistive load of class-E power amplifier. According to [25], $V^{\prime}$ has an amplitude of $V_{\text {om }}$ and phase of $\varphi=-$ $32.4^{0}$. The maximum possible value of $V_{\text {om }}$ is $1.074 V_{D D}=1.074 \times 12 \mathrm{~V}=12.888 \mathrm{~V}$, thus the voltage $V_{i}$ can be determined by

$$
V_{i}=\left(R_{1}+R_{r e f l}+j X\right) I_{1}=(1.7463+j 2) I_{1}
$$

Substituting all calculated mutual inductances, coil's self-inductances, coil's AC resistances, $X$, and $C_{5}$ into (26) and (27) gives

$$
\left[\begin{array}{l}
V_{i} \\
0 \\
0 \\
0 \\
0 \\
0 \\
0
\end{array}\right]=\left[\begin{array}{ccccccc}
0.185+j 2 & j 0.558 & j 0.034 & j 0.0064 & j 0.0006 & j 0.00055 & j 0.0005 \\
j 0.558 & 0.2 & j 0.3388 & j 0.0381 & j 0.0032 & j 0.0028 & j 0.0026 \\
j 0.034 & j 0.3388 & 0.27246 & j 0.289 & j 0.0149 & j 0.0121 & j 0.0098 \\
j 0.0064 & j 0.0381 & j 0.289 & 0.172 & j 0.411 & j 0.21 & j 0.1188 \\
j 0.0006 & j 0.0032 & j 0.0149 & j 0.411 & 0.07+j 8.9 & -j 2.972 & -j 3.793 \\
j 0.00055 & j 0.0028 & j 0.0121 & j 0.21 & -j 2.972 & 0.07+j 8.9 & -j 2.972 \\
j 0.0005 & j 0.0026 & j 0.0098 & j 0.1188 & -j 3.793 & -j 2.972 & 0.07+j 8.9
\end{array}\right]\left[\begin{array}{l}
I_{1} \\
I_{2} \\
I_{3} \\
I_{3} \\
I_{4} \\
I_{5} \\
I_{6} \\
I_{7}
\end{array}\right]
$$

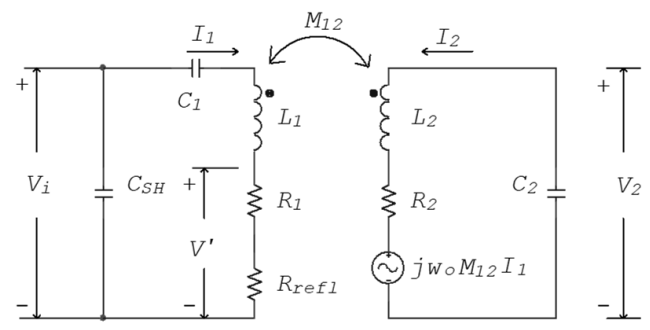

Figure 9. Modeling of the first WPT system (WPT1). 
Substituting (28) into (29) and determining the inverse of the resulted matrix produce $I_{2}=$ $(-0.136-j 1.61) I_{1}, I_{3}=(-0.8+j 0.341) I_{1}, I_{4}=(-0.306+j 1.184) I_{1}, I_{5}=(0.039-j 0.13) I_{1}, \quad I_{6}=$ $(0.031-j 0.101) I_{1}$, and $I_{7}=(0.033-j 0.101) I_{1}$. determined by

The output voltage $V_{0}$, input power $P_{i}$, output power $P_{0}$, and overall link efficiency $\eta_{\text {overall }}$ are

$$
\begin{aligned}
& V_{0}=-\left(I_{5}+I_{6}+I_{7}\right) \frac{1}{j \omega_{0} C_{5}}=(1.462+j 0.404) I_{1} \\
& P_{i}=I_{1}^{2}\left(R_{1}+R_{r e f l}\right)=1.7463 I_{1}{ }^{2} \\
& P_{0}=\frac{V_{0}^{2}}{R_{L}}=\frac{\left|(1.46212+j 0.404) I_{1}\right|^{2}}{R_{L}}=\frac{2.3 I_{1}{ }^{2}}{R_{L}} \\
& \eta_{\text {overall }} \%=\frac{P_{0}}{P_{i}} \times 100 \%=\frac{1.32}{R_{L}} \times 100 \%
\end{aligned}
$$

The above overall link efficiency corresponds to wirelessly energizing a load circuit located at $6.61 \mathrm{~m}$ from the power source taking into account strengthening the inductive coupling environment and conditioning the power receiving circuit for maximum power reception. If only one receiving coil is used and located at the same distance from the power source without using resonators for strengthening the inductive coupling, then according to (13), the efficiency for single coil receiving circuit without using resonators is calculated at $R_{L}=130 \Omega$ as follows:

$$
\eta_{\text {single coil }} \%=\frac{Q_{1}\left(Q_{5}\right)^{2} k_{15}^{2}}{\omega_{0} L_{5} Q_{L}} \times 100 \%=0.00421 \%
$$

The overall link efficiency of the proposed system at a load of $130 \Omega$ is calculated as

$$
\eta_{\text {overall }}=\frac{1.32}{R_{L}} \times 100 \%=\frac{1.32}{130} \times 100 \%=1.0154 \%
$$

To show the significance of the efficiency enhancement processed in this work, what is known as the efficiency gain $G_{\text {eff }}$ adopted here reflects this as follows:

$$
G_{\text {eff }}=\frac{1.0154}{0.00421}=241.184
$$

Many techniques are exploited in the design of the WPT transmitter to target relatively remote electrical nodes required to be wirelessly energized. The most efficient technique is class-E power amplifier, which is a switch mode power amplifier characterized by high efficiency and high power. Therefore such kind of power amplifiers are recommended to be adopted in the design of WPT systems. Figure 10 shows the PSpice design of the proposed efficient WPT system.

The class-E power amplifier in the proposed system is loaded by an RLC combination composed of a series RLC circuit represented by $C_{l}, L_{l}$, and $R_{l}$ shunted by a capacitor $C_{S H} . L_{l}$ and $R_{l}$ represent the inductance and $\mathrm{AC}$ resistance of the transmitting coil, respectively. $C_{l}$ is a tuning capacitor resonates with $L_{l}$ at a frequency slightly less than $f_{0}$, whereas $C_{S H}$ resonates with the series $\operatorname{RLC}\left(C_{l}, L_{l}\right.$, and $\left.R_{l}\right)$ circuit at $f_{0}$. Using (22) at an operating frequency of $338.6 \mathrm{kHz}, C_{l}$ is calculated as $4.8631 \mathrm{nF}$. $C_{S H}$ is determined by [25]

$$
C_{S H}=\frac{0.1936}{\omega_{0} R}=52.1 n F
$$




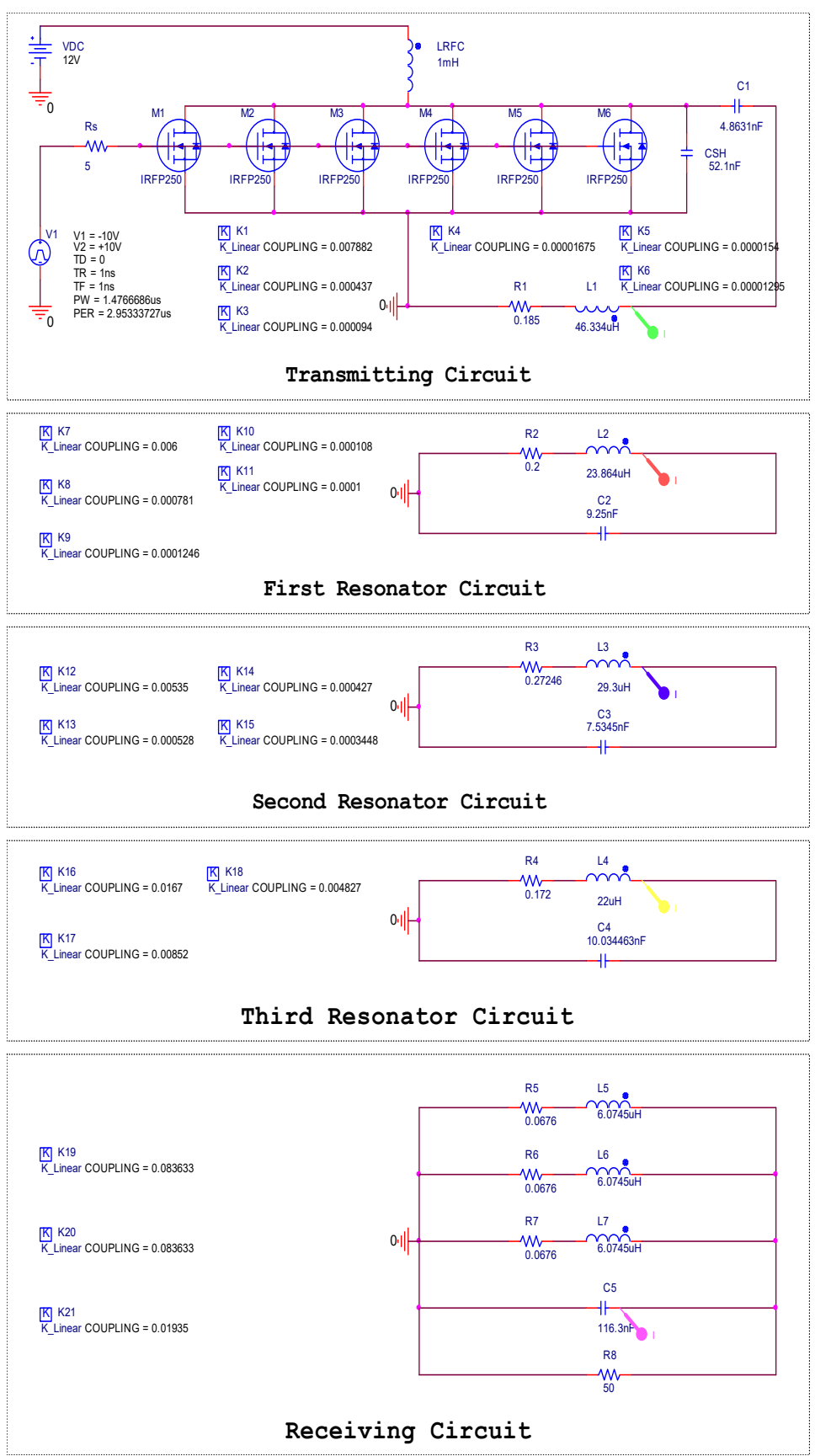

Figure 10. The PSpice design of the proposed system.

\section{RESULTS AND DISCUSSION}

The proposed efficient WPT system was first designed on PSpice, tested, and then practically implemented, thus in this work, there are simulative results, which are validated by experimental results.

\subsection{Simulation results}

The MOSFET triggering voltage signal $V_{S}$ is a square wave voltage having a $338.6 \mathrm{kHz}$ frequency and $\pm 10 \mathrm{~V}$ voltage levels. The DC current $I_{D C}$ of the system power amplifier and the output received voltage $v_{o}$ during $50 \Omega$ resistance are shown in Figure 11(a). The DC input current is about 2.5A and the output received $\mathrm{AC}$ voltage is about $5.36 \mathrm{~V}$ peak value. The system input power is $I_{D C} V_{D C}$, which is equal to 
$2.5 \mathrm{~A} \times 12 \mathrm{~V}=30 \mathrm{~W}$, while the received output $\mathrm{AC}$ power can be calculated as $0.5\left(v_{o p}\right)^{2} / R_{L}=0.5(5.36 \mathrm{~V})^{2} / 50 \Omega=$ $0.29 \mathrm{~W}$. Here $v_{o p}$ represents the peak value of $v_{o}$. Thus the overall system efficiency can be calculated as $(0.29 \mathrm{~W} / 30 \mathrm{~W}) \times 100 \%=0.967 \%$. Figure 11 (b) shows the AC currents $i_{1}, i_{2}, i_{3}$, and $i_{4}$, which are respectively corresponding to transmitting coil, first, second, and third resonator's currents during loading the proposed WPT system with $50 \Omega$ resistance. The results reveal the peak values of the AC currents $i_{1}, i_{2}, i_{3}$, and $i_{4}$ of 8.6A, 10.81A, 6.29A, and 5.2A, respectively. Figure 11(c) shows the receiving coil's currents $i_{5}, i_{6}$, and $i_{7}$, in addition to the receiving tuning capacitor current $i_{8}$ during loading the proposed WPT system with $50 \Omega$ resistance. The results reveal peak values of the AC currents $i_{5}, i_{6}$, and $i_{7}$ of $0.516 \mathrm{~A}, 0.4085 \mathrm{~A}$, and $0.409 \mathrm{~A}$, respectively. Figure $11(\mathrm{~d})$ shows the $\mathrm{AC}$ voltages $v_{1}, v_{2}, v_{3}$, and $\mathrm{v}_{4}$ which are respectively corresponding to the transmitting coil, first, second, and third resonators during loading the proposed WPT system with $50 \Omega$ resistance. The results reveal peak values of the AC voltages $v_{1}, v_{2}, v_{3}$, and $v_{4}$ of $858.4 \mathrm{~V}, 550 \mathrm{~V}, 390 \mathrm{~V}$, and $240 \mathrm{~V}$, respectively.

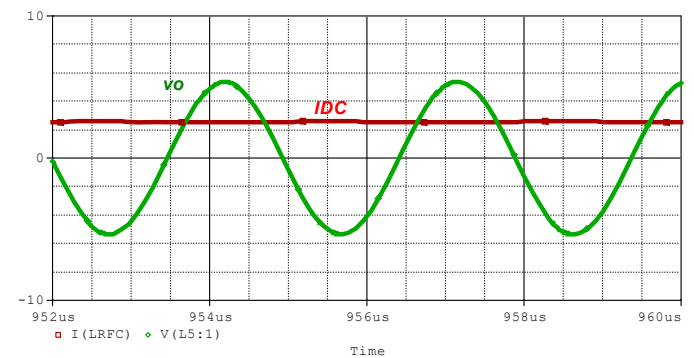

(a)

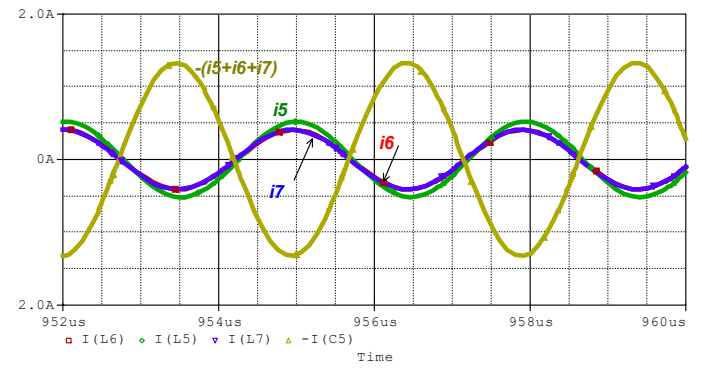

(c)

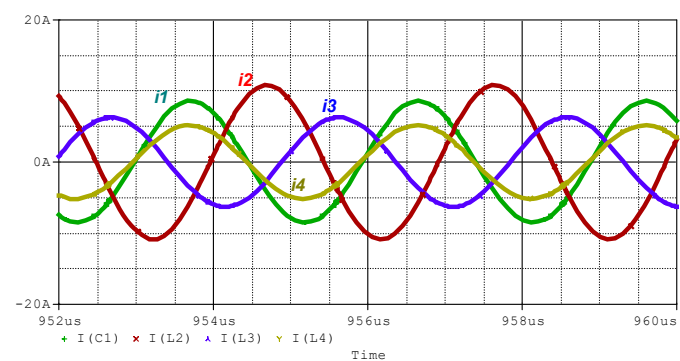

(b)

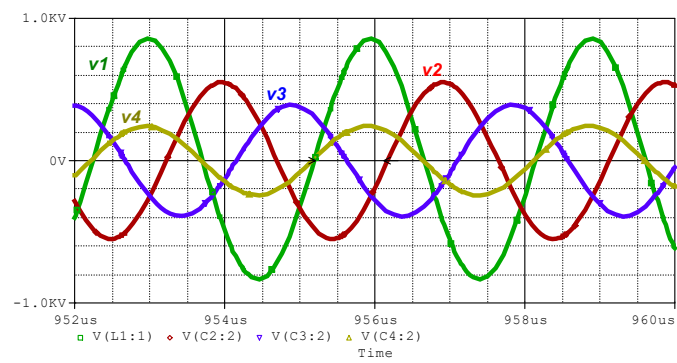

(d)

Figure 11. The proposed WPT system currents and voltages during $50 \Omega$ load. (a) DC input current and the received AC voltage, (b) the transmitting coil, first, second, and third resonator's currents, (c) the transmitting coil, first, second, and third resonator's voltages, (d) the receiving currents.

The overall WPT system was extra tested at different loading conditions Table 4 summarizes the efficiency changes as load resistance varied from $12.5 \Omega$ to $125 \Omega$, while Figure 12 shows a graph reflecting the efficiency changes with load variations.

Table 4. Coil's calculated AC resistance

\begin{tabular}{cc}
\hline Load Resistance $(\Omega)$ & System Efficiency\% \\
\hline 12.5 & 0.973 \\
25 & 1.07 \\
30 & 1.14 \\
35 & 1.1 \\
50 & 0.967 \\
75 & 0.72 \\
100 & 0.6 \\
125 & 0.5 \\
\hline
\end{tabular}




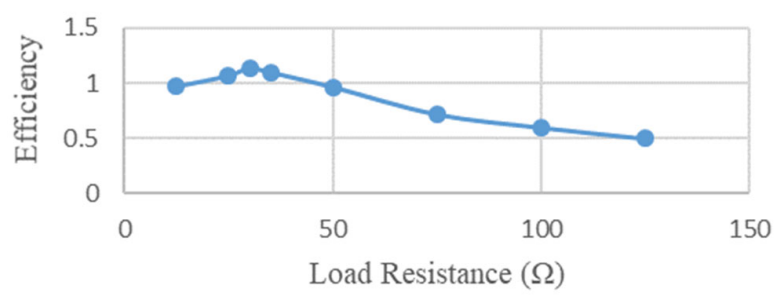

Figure 12. The efficiency of the proposed efficient WPT system against load resistance

\subsection{Experimental results}

The results introduced here are reflecting the performance of the whole proposed system during energizing a $50 \Omega$ resistive load, lighting a LED, and charging $1.2 \mathrm{~V}$ rechargeable battery. Figure 13(a) shows the oscilloscope reading of the transmitting coil and first resonator voltages, which have peak to peak values of $900 \mathrm{~V}$ and $280 \mathrm{~V}$, respectively. The oscilloscope voltage gain for both channel are $10 \mathrm{~V} / \mathrm{cm}$. The yellow reading corresponds to transmitter voltage, while the blue one corresponds to first resonator voltage. Figure 13(b) shows the oscilloscope reading of the second coil and third resonator voltages, which have peak to peak values of $188 \mathrm{~V}$ and $96 \mathrm{~V}$, respectively. The oscilloscope voltage gain for both channel are $10 \mathrm{~V} / \mathrm{cm}$ and the yellow reading corresponds to first resonator voltage, while the blue corresponds to second resonator voltage. Figure 13(c) shows the oscilloscope reading of the receiving circuit voltage, which has peak to peak value of $2.56 \mathrm{~V}$ during energizing $50 \Omega$ resistive load.

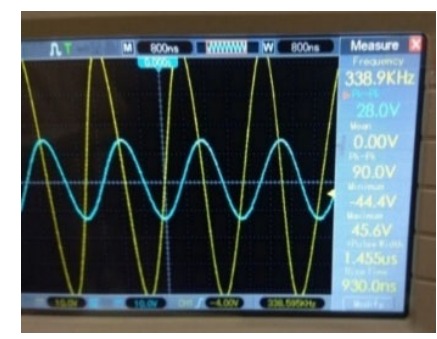

(a)

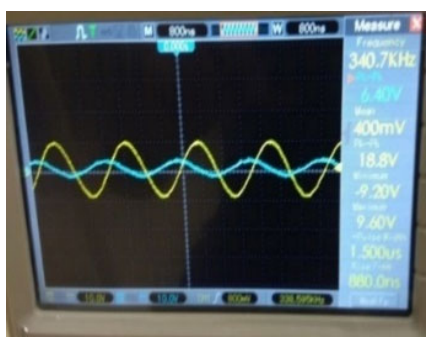

(b)

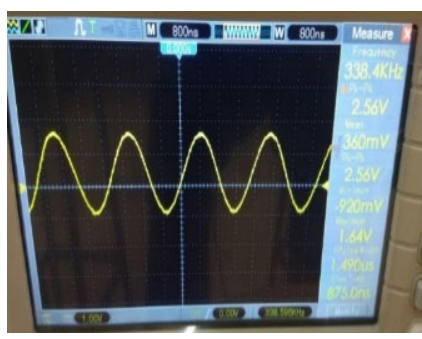

(c)

Figure 13. The AC voltages of (a) the transmitting coil and first resonator, (b) second and third resonators, (c) receiving circuit during loading the proposed WPT system with $50 \Omega$ resistance.

Figure 14(a) shows the oscilloscope reading of the receiving circuit voltage of $4.8 \mathrm{~V}$ peak to peak during lighting a LED shown in Figure 14(b).

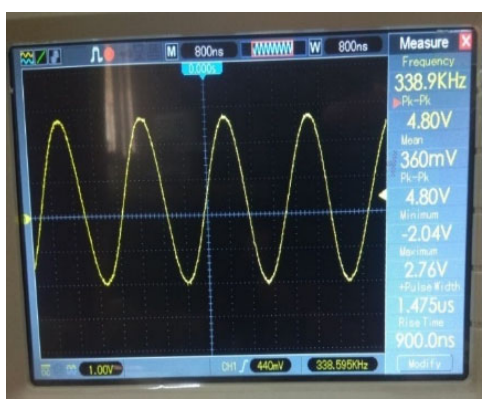

(a)

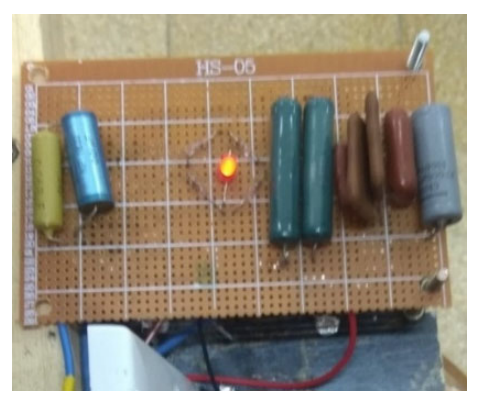

(b)

Figure 14. (a) The received voltage of the proposed WPT system during lighting a LED, (b) LED circuit. 
Figure 15(a) shows the oscilloscope reading of the receiving circuit voltage, which has peak to peak value of $4.28 \mathrm{~V}$ during charging a $1.2 \mathrm{~V}$ rechargeable battery shown in Figure 15(b).

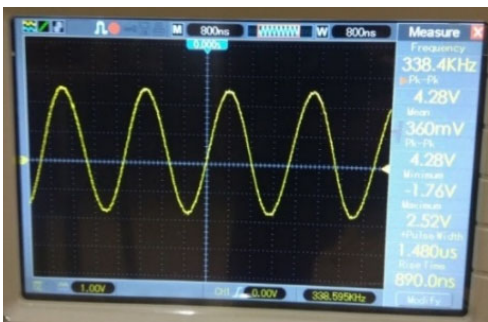

(a)

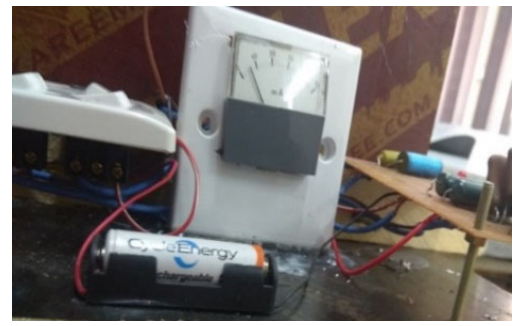

(b)

Figure 15. (a) The received voltage of the WPT system during charging $1.2 \mathrm{~V}$ battery, (b) charging circuit.

Figure 16 shows the oscilloscope reading of the voltage of a receiving circuit having a single coil instead of three coils during $50 \Omega$ load. The oscilloscope reading is $1.28 \mathrm{~V}$ peak to peak value.

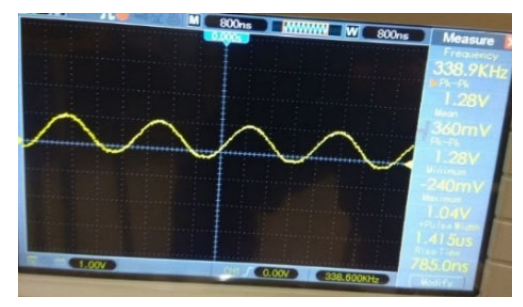

Figure 16. The voltage of a single coil receiving circuit in the proposed WPT system during energizing $50 \Omega$ resistive load.

Figure 17 shows voltage and current readings of the DC power supply. The voltage reading is $12 \mathrm{~V}$ $\mathrm{DC}$, while the current reading is $1.9 \mathrm{~A}$ DC. The input DC power is $P_{i}=I_{D C} V_{D C}=1.9 \mathrm{~A} \times 12 \mathrm{~V}=22.8 \mathrm{~W}$, while the AC received power $P_{0}$ for three coil receiving circuit is calculated as $P_{0}=0.25\left(v_{\text {opp }}\right)^{2} / \mathrm{RL}=0.25(2.56 \mathrm{~V})^{2} / 50 \Omega=0.032768 \mathrm{~W}$. Therefore, the overall system efficiency is calculated as $\eta_{\text {overall }}=\left(P_{0} / P_{i}\right) \times 100 \%=0.144 \%$. The received output power for single coil receiving circuit is $0.25(1.28 \mathrm{~V})^{2} / 50 \Omega=0.008192 \mathrm{~W}$. The system efficiency for single coil receiving circuit is $0.036 \%$. Therefore, the proposed WPT system with three parallel connected receiving coils has an overall efficiency four times greater than that of a same system having single receiving coil. This means utilizing parallel connected coils in the receiving circuit significantly enhances the overall system efficiency.

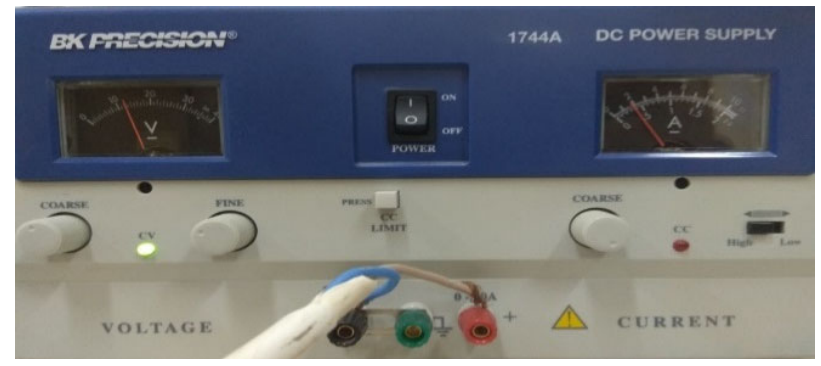

Figure 17. The DC power supply voltage and current readings. 
The effects of resonators on received $\mathrm{AC}$ power are addressed in this work. Table 5 lists the received $\mathrm{AC}$ power and efficiency for all important cases of removing resonators from the circuit of the practical WPT system taking into account a DC input power of $22.8 \mathrm{~W}$, which is taken as reference power during the calculation of efficiency.

Table 5. Efficiency gain values of different WPT systems

\begin{tabular}{lcc}
\hline \multicolumn{1}{c}{ System Type } & Received AC Power (mW) & Efficiency \% \\
\hline Overall WPT System & 32.768 & 0.144 \\
Overall WPT System without First resonator & 1 & 0.02 \\
Overall WPT System without Second resonator & 3.53 & 0.075625 \\
Overall WPT System without Third resonator & 1.25 & 0.003 \\
Overall WPT system without second \& third resonators & 0.2 & $74.6 \times 10^{-5}$ \\
Overall WPT System without resonators & 0.0026 & $3 \times 10^{-5}$ \\
\hline
\end{tabular}

\section{CONCLUSION}

In this work, an adaptive efficient WPT system is introduced. This system is carried out on PSpice and validated experimentally. Both simulative and experimental WPT system have accomplished significant enhancement in efficiency. The proposed WPT systems has three resonators and three parallel connected identical receiving coils located at $6.61 \mathrm{~m}$ from the power transmitter. The efficiency enhancement approaches thousands times the efficiency of a conventional WPT system having similar power transmitter located at the same distance from the receiving circuit, which has a single coil identical to those in the proposed efficient WPT system. The conclusions of this work are summarized in: Both simulative and practical systems have demonstrated that efficiency enhancement can be accomplished through inserting resonators along with energy transmission path. Both simulative and practical systems have demonstrated that efficiency enhancement can be accomplished through the modification of the receiving circuit using parallel connected receiving coils. More power can be received when the reactance of the equivalent receiving coil is much less than the load impedance. Energy transmission can approach distant nodes via inserting more resonators along the path toward the targeted node. Heavier power transmission requires lower frequency range, but bigger sending and receiving antennas.

Both systems show similar responses, which they differ in amounts, but exhibit similar behaviors. The differences in amounts are due to commercial tuning capacitors which have dielectric resistances responsible for causing significant reduction in the resonant currents in all resonator circuits.

\section{REFERENCES}

[1] A. Esser and H.-C. Skudelny, "A new approach to power supplies for robots," IEEE Transactions on Industry Applications, vol. 27, no. 5, pp. 872-875, 1991.

[2] L. Cannon, J. F. Hoburg, D. D. Stancil, S. C. Goldstein, "Magnetic resonant coupling as a potential means for wireless power transfer to multiple small receivers," IEEE Transactions on Power Electronics, vol. 24, no. 7, pp. 1819-1825, 2009.

[3] J. J. Casanova, Z. N. Low, and J. Lin, "Design and optimization of a Class-E amplifier for a loosely coupled planar wireless power system," IEEE Transactions on Circuits and Systems-II: Express Briefs, vol. 56, no. 11, pp. 830834, 2009.

[4] J. A. Taylor, Z. N. Low, J. Casanova, and J. Lin, "A wireless power station for laptop computers Jason," IEEE Radio and Wireless Symposium (RWS), 10-14 Jan. 2010, New Orleans, LA, USA, pp. 625-628, 2010.

[5] M. Pinuela, D. C. Yates, S. Lucyszyn, and P. D. Mitcheson, "Maximizing DC to load efficiency for inductive power transfer," IEEE Transactions on Power Electronics, vol. 50, no. 6, pp. 1-11, 2010.

[6] A. Massa, G. Oliveri , F. Viani, and P. Rocca, "Array designs for long-distance wireless power transmission: Stateof-the-art and innovative solutions," Proceedings of the IEEE, vol.101, no. 6, pp. 1464- $1481,2013$.

[7] S. Raju, R. Wu, M. Chan, and C. P.Yue, "Modeling of mutual coupling between planar inductors in wireless power applications," IEEE Transactions On Power Electronics, vol. 29, no. 1, pp. 481-490, 2014.

[8] Y. Cheng and Y. Shu, "A new analytical calculation of the mutual inductance of the coaxial spiral rectangular coils," IEEE Transactions on Magnetics, vol. 50, no. 4, pp. 1-6, 2014.

[9] J. Kim, -J. Park, "Approximate closed-form formula for calculating ohmic resistance in coils of parallel round wires with unequal pitches," IEEE Transactions On Industrial Electronics, vol. 62, no. 6, pp. 3482-3489, 2015.

Int J Pow Elec \& Dri Syst, Vol. 11, No. 2, June 2020 : $711-725$ 
[10] L. Tan, J. Li, C. Chen, C. Yan, J. Guo, and X. Huang, "Analysis and performance improvement of wpt systems in the environment of single non-ferromagnetic metal plates," Energies, vol 9, pp. 1-16, 2016.

[11] V. V. Nair and J. R. Choi, "An efficiency enhancement technique for a wireless power transmission system based on a multiple coil switching technique," Energies vol. 9, pp. 1-15, 2016.

[12] J. Chen, Z. Ding, Z. Hu, Sh. Wang, Y. Cheng, M. Liu, B. Wei, and S. Wang, "Metamaterial-based high-efficiency wireless power transfer system at 13.56 Mhz for low power applications," Progress in Electromagnetics Research, vol.72, pp.17-30, 2017.

[13] X. Liu, T. Wang, X. Yang, N. Jin, and H. Tang, "Analysis and design of a wireless power transfer system with dual active bridges," Energies vol. 10, pp. 1-20, 2017.

[14] H.-D. Lang and C. D. Sarris, "Optimization of wireless power transfer systems enhanced by passive elements and metasurfaces," IEEE Transactions on Antennas and Propagation, vol. 6, no. 10, pp. 5462- 5474, 2017.

[15] M. Schormans, V. Valente, and A. Demosthenous, "Practical inductive link design for biomedical wireless power transfer: A tutorial," IEEE Transactions on Biomedical Circuits and Systems, vol. 12, no. 5, pp. 1112 - 1130, 2018.

[16] S. Liu, J. Su, and J, Lai, "Accurate expressions of mutual inductance and their calculation of archimedean spiral coils," Energies vol 12, 2017.

[17] A. Kurs, "Power transfer through strongly coupled resonances," Master Thesis, Massachusetts Institute of Technology, Massachusetts Ave, Cambridge, USA 2008.

[18] T. Peng, S. Koulouridis, and J. L. Volakis, "Miniaturization of conical helical antenna via optimized coiling," Aces Journal, vol. 26, no. 6, pp. 452-458, 2011.

[19] W. Junhua, "Resonant coupling energy transmission technology and its applications to implanted medical devices," $\mathrm{PhD}$ Thesis, The Hong Kong Polytechnic University, Hong Kong, 2012.

[20] M. C. Pickelsimer, "Wireless power transfer system for electric vehicles with power factor correction," MSc Thesis, University of Tennessee, Knoxville, Tennessee, 2012.

[21] A. Bourřa and M. Husák, "Communication and powering scheme for wireless and battery-less measurement," Radioengineering, vol. 21, no. 1, pp. 239-245, 2012.

[22] T. Sun, X. Xie, and Z. Wang, "Wireless power transfer for medical microsystems", Springer, New York, USA, 2013.

[23] E. B. Wasatonic, "Comparison of tesla coil driver topologies: Rotary spark gap versus double resonant solid state," MSc Thesis, The Pennsylvania State University, Pennsylvania, USA, 2013.

[24] R. Ludwig and P. Bretchko, "RF circuit design: Theory \& applications," Prentice Hall, Upper Saddle River, New Jersey, USA, 2000.

[25] J. Rogers and C. Plett, "Radio frequency integrated circuit design," Artech House, Boston, London, U.K, 2003. 